\title{
Letter From the Guest Editor
}

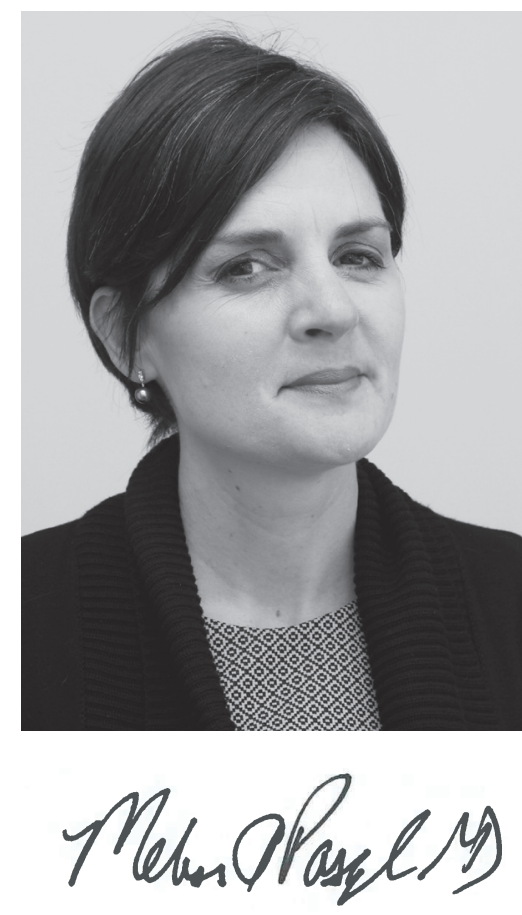

Melissa A. Pasquale-Styles MD Guest Editor

Since the term sudden infant death syndrome (SIDS) was first used in the 1960's, sudden infant deaths of undetermined cause continue to represent a leading cause of infant mortality in the United States. In a majority of these, the underlying etiology cannot be identified in the autopsy room, under a microscope, or in postmortem toxicology. Yet, the careful, complete autopsy remains essential to proving that the answer lays elsewhere. It also allows for preservation of samples for potential future analysis. Research has shown that there are strong independent risk factors for sudden infant death, including but not limited to an unsafe sleep environment, history of prematurity, low birth weight, lack of prenatal care, maternal smoking, and poor socioeconomic status. Consistent racial and gender disparities persist that are not well understood. As we continue to work toward improved, standardized infant death investigations and explore the frontiers of molecular genetics and neurobiology, it is increasingly necessary to clearly communicate what we know and what we don't know about sudden infant death.

Sudden infant death syndrome served for a period of time as useful terminology to identify a cohort for research and provide some context for families. It has traditionally been assigned a natural manner of death in most jurisdictions where used. However, its utility disintegrated when better scene investigations showed that most infants die in unsafe sleep environments. Sudden infant death syndrome cannot effectively encompass both natural and unnatural manners of deaths, and many infant deaths are known or suspected to be caused by unsafe sleep-related asphyxia. At times it is impossible to say if the infant died in an unsafe sleep environment or because of the unsafe sleep environment. In some cases, it is possible that- 
there are other inherent vulnerabilities that may not be measurable and may contribute to the death. While asphyxia in an unsafe sleep environment is not the final answer to the mystery of all sudden infant deaths, it is a specific cause that is important to identify because it can be targeted for prevention with proven effect, demonstrated by the impact of the "Back to Sleep" campaign. Where there is reasonable certainty based on the death investigation, medical examiners and coroners should certify a death as asphyxia. If the cause of death cannot be determined by a complete autopsy and scene investigation, then it is more scientifically accurate to certify a death as undetermined cause and manner.

Regardless of how deaths are certified in each jurisdiction, the burden is on medical examiners and coroners to explain the reason behind the certification to affected parties, starting with the individual families in each case. This is most important when the cause and manner are undetermined, especially if preventable risks such as an unsafe sleep environment cannot be ruled out as a contributing factor. It is equally important for medical examiners and coroners to maintain an open dialogue about local infant death certification practice with epidemiologists at local, state, and federal levels who struggle to understand regional variances in certification in order to drive research and prevention strategies. Similarly, supportive organizations dedicated to awareness and prevention of sudden infant death can be powerhouses for education and research funding, but they need reliable information from the medicolegal death investigation system to help guide their missions.

Infant death investigations often leave us with more questions than answers, but history has shown that there is no scientific reason to believe we are searching for a singular etiology to explain a syndromic disorder. In the quest to identify the many various causes of sudden infant deaths, SIDS has become outdated terminology that now serves only as a red herring. 